\title{
Investigação do potencial antioxidante e anticolinesterásico de 20 espécies da família Lauraceae
}

\author{
Klenicy Kazumy de Lima YAMAGUCHI ${ }^{1}$, Joelma Moreira ALCÂNTARA ${ }^{1}$ e \\ Valdir Florêncio da VEIGA JUNIOR ${ }^{1}$
}

\section{RESUMO}

Lauraceae é uma das famílias mais importantes na floresta amazônica. Diversas de suas espécies apresentam em sua composição substâncias com pronunciadas atividades biológicas. Nesse trabalho, os extratos obtidos em etanol de galhos e folhas de 20 espécies de Lauraceae foram coletados na Reserva Florestal Ducke e avaliados quanto às atividades antioxidante frente ao radical livre 2,2-difenil-1-picrilhidrazila(DPPH) e inibidora da enzima acetilcolinesterase. O extrato dos galhos de Licaria martiniana apresentou a maior capacidade de sequestro do radical DPPH $\left(6,96 \mu \mathrm{g} \cdot \mathrm{mL}^{-1}\right)$, mas os extratos de Ocotea minor foram os mais ativos na inibição da enzima acetilcolinesterase, apresentando também pronunciada atividade antioxidante. O perfil de atividade antioxidante e anticolinesterásico destes extratos está sendo relatado pela primeira vez neste trabalho.

PALAVRAS-CHAVE: Lauraceae, DPPH, anticolinesterásico, Licaria martiniana, Ocotea minor.

\section{Antioxidant and anticholinesterasic effects of 20 species of the family Lauraceae}

\section{ABSTRACT}

Lauraceae is one of the most important families in the Amazon rainforest. Several substances with strong pharmacological properties have been isolated from the species of this family. In this study, ethanol extracts of branches and leaves of 20 Lauracea especies were collected in the Reserva Florestal Ducke and evaluated for antioxidant activity of extracts using the 2,2-diphenyl1-picrylhydrazyl (DPPH) and inhibition of acetylcholinesterase. The branches extracts from Licaria Martiniana showed the highest capacity to scavenge the DPPH radical $\left(6.96 \mu \mathrm{gmL}^{-1}\right)$, butOcotea minor extracts were the most active in inhibiting the enzyme acetylcholinesterase, with also pronounced antioxidant activity. The antioxidant and acetylcholinesterasic activities profile of these extracts is being reported in the first time in this paper.

KEYWORDS: Lauraceae, DPPH, antiacetylcholinesterase, Licaria martiniana, Ocotea minor. 


\section{INTRODUÇÃO}

Com o aumento da expectativa de vida da populaçáo mundial, tornam-se cada vez mais frequentes as doenças relacionadas à idade, tal como a doença de Alzheimer (DA). A DA é neurodegenerativa, progressiva e que afeta principalmente a população idosa. Suas causas ainda não são determinadas e, assim, até o momento somente os sintomas podem ser amenizados (Quik et al. 1999; Viegas Junior et al. 2004).

Os avanços obtidos na compreensão da evolução da doença têm demonstrado que o uso de inibidores da enzima acetilcolinesterase (AChE) é a forma mais eficiente de controle da doença. Além disso, as espécies reativas de oxigênio e nitrogênio atualmente são consideradas fatores contribuintes para o desencadeamento desta patologia no organismo (Houghton et al. 2007). O medicamento mais efetivo na inibição da AChE, utilizado no tratamento da DA, é o alcaloide galantamina, isolado inicialmente de Galanthus woronowii (López et al. 2002).Apesar de ser o melhor tratamento disponível atualmente, esta substância apresenta diversos efeitos colaterais e preço elevado no mercado. A busca por novos fármacos que sejam menos tóxicos, mais efetivos no controle da enzima AChE e com menor valor de mercado, torna-se uma necessidade.

Ensaios de varredura são ferramentas muito utilizadas para detectar a atividades biológicas em grande quantidade de amostras e com baixo custo, incluindo o uso de sistemas robotizados, como o High Throughput Screening (HTS). Sua utilização reduz em muito o tempo de análise tradicional em especial nos extratos vegetais, que normalmente envolvem o fracionamento e purificação exaustiva de substâncias. Ensaios de varredura de inibição de $\mathrm{AChE}$ em extratos de plantas com o objetivo de selecionar apenas espécies promissoras para que possam haver estudos fitoquímicos bioguiados é uma estratégia inteligente para diminuir o tempo e o custo das análises.

Os trabalhos de Berkov et al.(2008) e Barbosa Filho et al. (2006) associam a ação de inibir a enzima acetilcolinesterase das espécies estudadas de diferentes famílias com a presença de alcaloides, devido ao fato de que a maioria das substâncias que apresentam atividade inibidora de acetilcolinesterase serem alcaloides. Dessa forma, as famílias detentoras desta classe de metabólitos tornam-se uma fonte promissora para a utilização de métodos para analisar esta atividade.

A família Lauraceae é uma das mais conhecidas por apresentar na composiçáo química de suas espécies a presença de alcaloides.Cordell et al.(2001) registraram em seu trabalho o isolamento de 425 alcaloides distribuídos em 189 espécies de 25 gêneros da família Lauraceae. Essas substâncias são na maioria dos tipos aporfínicos e geralmente apresentam excelentes atividades biológicas, como: a nantenina, um bloqueador de contração muscular e translocaçáo de $\mathrm{Ca}^{2+}$ (Ribeiro et al. 2003), e seus derivados atuam como antagonistas $\alpha^{1}$-adrenoreceptor (Indra et al. 2002); a coclaurina, uma substância com ação anti-HIV (Kashiwada et al. 2005)e derivados halogenados da predicentrina que inibem a topoisomerase II (Woo et al. 1999).

A família Lauraceae possui aproximadamente 50 gêneros e 2500 espécies, sendo que no Brasil existem cerca de 400 espécies distribuídas em 25 gêneros (Cronquist 1981; Burger e Van der Werff 1990; Chanderbali et al. 2001). Apresenta importância mundial, como fonte de produtos naturais de alto valor comercial, com espécies utilizadas em grande escala como o abacate (Persea americana), a canela (Cinnamomum verum) e espécies aromáticas com óleos utilizados para perfumaria como os extraídos de canela-sassafrás (Ocotea odorifera) e pau rosa (Aniba rosaeodora) (Quinet e Andreata 2002; Marques 2001). Muitas espécies desta família encontradas no Brasil ainda não foram investigadas quanto às suas atividades biológicas.

O presente trabalho teve como objetivo realizar uma varredura para as atividades antioxidante, pelo método de DPPH, e anticolinesterásica dos extratos em etanol de 20 espécies de Lauraceae encontradas na Reserva Florestal Ducke, de ocorrência na Floresta Amazônica brasileira distribuídas em nove gêneros: Aniba, Dicypellium, Endlicheria,Licaria, Mezilaurus, Ocotea, Pleurothyrium, Rhodostemonodaphne e Sextonia.

\section{MATERIAL E MÉTODOS}

\section{MATERIAL VEGETAL}

As amostras foram coletadas na Reserva Florestal Ducke, Manaus, Amazonas em três épocas distintas: 1) março de 2007 e 2008: Aniba panurensis (INPA 177317), Aniba rosaeodora (INPA 177315),Dicypellium manausense (INPA 210723), Licaria cannella angustata (INPA 177340), Licaria martiniana (INPA 177312), Mezilaurus duckei(INPA 181931), Mezilaurus itauba(INPA 177309), Ocotea nigrescens(INPA 177346), Ocotea splendens(INPA 177318), Rhodostemonodaphne negrensis(INPA 177320), Rhodostemonodaphne parvifolia(INPA 189875), Pleurothyrium vasquezii(INPA 181828)e Sextonia rubra (INPA 177257); 2) agosto de 2009:Endlicheria citriodora(INPA 191998), Endlicheria sericea(INPA 177323), Ocotea ceanothifolia(INPA 189734), Ocotea leucoxylon(INPA 177304), Ocotea minor(INPA 189746), Rhodostemonodaphne crenaticupula(INPA 185563)e Rhodostemonodaphne recurva(INPA 177326). As exsicatas das espécies encontramse depositadas no herbário do Instituto Nacional de Pesquisas da Amazônia (INPA) e foram identificadas no Projeto Flora da Reserva Ducke (Ribeiro et al. 1999). 


\section{OBTENÇÃO DOS EXTRATOS}

O material vegetal foi seco, moído e extraído por maceração a frio durante 72 horas em etanol previamente destilado. Após a maceração e filtração, o solvente foi eliminado em evaporador rotatório, resultando num extrato bruto. O material vegetal foi recolocado em maceração, repetindo-se o processo descrito anteriormente durante quatro vezes, obtendo assim um extrato em etanol.

\section{ATIVIDADE ANTIOXIDANTE QUALITATIVA}

Os extratos foram avaliados com relação a sua capacidade de sequestro do radical livre estável 2,2'-difenil-1-picrilhidrazila (DPPH), por Cromatografia em Camada Delgada (CCD) segundo método de Soler-Rivas et al. (2000). Foram aplicados $10 \mu \mathrm{L}$ de cada solução do extrato e da substância de referência [quercetina dissolvida em metanol $\left(1 \mathrm{mg} \cdot \mathrm{mL}^{-1}\right)$ ] sobre a cromatoplaca.

Após a evaporaçáo do solvente a placa foi nebulizada com a solução de DPPH. Depois de trinta segundos foi realizada a leitura da placa, em que halos amarelados sobre fundo de coloraçáo púrpura, foram considerados positivos.

\section{ATIVIDADE ANTIOXIDANTE QUANTITATIVA}

A atividade antioxidante foi avaliada por meio da reação de redução do radical livre 1,1-difenil-2-(2,4,6-trinitrofenil) hidrazila $(\mathrm{DPPH} \bullet)$, que em contato com substâncias antioxidantes é reduzido a hidrazina, segundo o método de Mensor (2001) adaptado em microplaca, utilizando quercetina como padrão. As soluçôes foram preparadas em metanol, para cada extrato analisado, com concentraçóes de 5 a $250 \mu \mathrm{g} \cdot \mathrm{mL}^{-1}$. $\mathrm{O}$ mesmo procedimento foi realizado para a soluçấo padrão com concentraçấo de 0,313 a $5 \mu \mathrm{g} \cdot \mathrm{mL}^{-1}$.

A leitura da absorbância das amostras foi realizada após 30 minutos da adição da solução de DPPH $(0,3$ mmol.L-1 $)$ em espectrofotômetro UV-Vis (modelo Biomat 3550 da Thermo Electron Corporation) no comprimento de onda $517 \mathrm{~nm}$, em microplaca de 96 poços.

A porcentagem de atividade antioxidante (\%AA) foi determinada a partir dos valores de absorbância de todas as concentraçóes testadas no tempo de $30 \mathrm{~min}$, conforme a equação:

$\% \mathrm{AA}=\left\{\left[\mathrm{Abs}_{\text {controle }}-\left(\mathrm{Abs}_{\text {amostra }}-\mathrm{Abs}_{\text {branco }}\right)\right] \times 100\right\} / \mathrm{Abs}_{\text {controle }}$ onde Abscontrole e Absamostra representam as absorbâncias da solução metanólica de DPPH e da mistura reacional (amostra + DPPH), respectivamente.

A concentração eficiente, quantidade de antioxidante necessária para decrescer a concentração inicial de DPPH em $50 \%\left(\mathrm{CE}_{50}\right)$, foi determinada através da regressão linear dos valores obtidos utilizando o programa Microsoft Office Excel
2007. Os resultados apresentados neste estudo correspondem à média de três repetiçóes $(\mathrm{n}=3) \pm$ desvio padrão da média.

\section{ATIVIDADE INIBIDORA DE ACETILCOLINESTERASE}

A inibição da enzima acetilcolinesterase (AChE) foi avaliada seguindo a metodologia descrita por Elmann et al. (1961), adaptada por Rhee et al. (2001). Este bioensaio consiste na aplicaçáo da amostra em cromatoplaca de camada delgada, seguida da pulverização da placa com o reagente de Ellman ácido 5,5'-ditiobis-[2-nitrobenzóico] e uma solução de iodeto de acetiltiocolina em tampão e deixa secar. Após este procedimento, pulveriza-se a placa com a enzima AChE (3 U. $\left.\mathrm{mL}^{-1}\right)$. A inibição enzimática pode ser constatada pela ausência da cor amarela e concomitante surgimento de um halo branco. Como padráo positivo de atividade foi utilizado o alcaloide eserina.

\section{RESULTADOS E DISCUSSÃO}

O teste com DPPH é um método bastante conveniente para a varredura da atividade antioxidante de pequenas moléculas, uma vez que a reação pode ser observada visualmente utilizando-se uma cromatoplaca ou espectrofotômetro UV/ VIS (Wagner e Bladt 1996). Os resultados da atividade antioxidante correspondem à quantidade necessária para decrescer a concentraçáo inicial de DPPH em 50\%, denominado, concentração eficiente $\left(\mathrm{CE}_{50}\right)$. Quanto maior o consumo de DPPH por uma amostra, menor será a sua $\mathrm{CE}_{50}$, como é o caso do padráo quercetina, com concentraçáo eficiente de $4,13 \mu \mathrm{g} \cdot \mathrm{mL}^{-1}$.

Verifica-se a partir dos testes qualitativos que os resultados dos ensaios de atividade antioxidante empregados não apresentaram relaçáo direta com os resultados obtidos nos testes de atividade anticolinesterásica. Os resultados dos testes químicos e biológicos realizados encontram-se sumarizados na Tabela 1.

Os galhos das espécies $O$. minor e $L$. martiniana foram os únicos que apresentaram atividades relevantes em ambos os ensaios, antioxidante e inibidor da enzima acetilcolinesterase, conforme a correlação encontrada por Traykova et al. (2003) para o alcaloide galantamina hidrobromida. As demais espécies não apresentaram resultados que puderam ser correlacionados nestes ensaios. No trabalho de Giordani $e t$ al. (2008) os resultados positivos para atividade antioxidante também não corresponderam com a inibição da enzima acetilcolinesterase.

Todos os extratos apresentaram potencial antioxidante no ensaio qualitativo, exceto os extratos das espécies $O$. splendens, $R$. negrensis, $R$. parvifolia e $P$. vasquezii.

Os resultados para $\mathrm{CE}_{50}$ variaram de $168,73 \pm 21,80$ a $6,96 \pm 0,13$. A atividade antioxidante foi maior para os extratos de E. sericeae $\left(\mathrm{CE}_{50}\right.$ de $\left.9,77 \mu \mathrm{g} \cdot \mathrm{mL}^{-1}\right), L$. martiniana $(6,96$ 
Tabela 1 -Atividades acetilcolineterásica e antioxidante qualitativa e quantitativa $\left(\mathrm{CE}_{50} \pm \mathrm{DP}\right)$ do extrato em etanol de vinte espécies de Lauraceae.

\begin{tabular}{|c|c|c|c|c|}
\hline Espécie botânica & Órgão & AChE qualitativo & Antioxidante qualitativo & $\mathrm{CE}_{50} \pm \mathrm{DP}\left(\mu \mathrm{g} \cdot \mathrm{mL}^{-1}\right)$ \\
\hline \multirow{2}{*}{ Aniba panurensis } & Folhas & - & + & $14,370 \pm 0,096$ \\
\hline & Galhos & + & + & $27,586 \pm 2,199$ \\
\hline Aniba rosaeodora & Galhos & - & + & $25,209 \pm 0,186$ \\
\hline \multirow{2}{*}{ Dicypellium manausense } & Folhas & - & + & $23,670 \pm 1,475$ \\
\hline & Galhos & - & + & $16,814 \pm 0,486$ \\
\hline \multirow{2}{*}{ Endlicheria citriodora } & Folhas & + & + & $161,01 \pm 1,00$ \\
\hline & Galhos & - & + & $42,78 \pm 0,53$ \\
\hline \multirow{2}{*}{ Endlicheria sericea } & Folhas & - & + & $9,77 \pm 0,42$ \\
\hline & Galhos & - & + & $50,92 \pm 0,96$ \\
\hline \multirow{2}{*}{$\begin{array}{l}\text { Licaria cannella } \\
\text { angustata }\end{array}$} & Folhas & - & + & $15,029 \pm 0,361$ \\
\hline & Galhos & + & + & $29,349 \pm 0,245$ \\
\hline \multirow{2}{*}{ Licaria martiniana } & Folhas & - & + & $11,168 \pm 1,416$ \\
\hline & Galhos & + & + & $6,956 \pm 0,131$ \\
\hline \multirow{2}{*}{ Mezilaurus duckei } & Folhas & - & + & $23,253 \pm 0,556$ \\
\hline & Galhos & - & + & $13,494 \pm 0,484$ \\
\hline Mezilaurus itauba & Folhas & - & + & $10,739 \pm 0,240$ \\
\hline \multirow{2}{*}{ Ocotea ceanothifolia } & Folhas & - & + & $18,12 \pm 0,18$ \\
\hline & Galhos & + & + & $15,64 \pm 0,72$ \\
\hline \multirow{2}{*}{ Ocotea leucoxylon } & Folhas & - & + & $52,51 \pm 1,42$ \\
\hline & Galhos & + & + & $50,88 \pm 0,64$ \\
\hline \multirow{2}{*}{ Ocotea minor } & Folhas & + & + & $8,21 \pm 0,66$ \\
\hline & Galhos & + & + & $9,08 \pm 0,51$ \\
\hline Ocotea nigrescens & Folhas & - & + & $168,726 \pm 21,798$ \\
\hline Ocotea splendens & Folhas & - & - & NR \\
\hline Pleurothyrium vasquezii & Folhas & - & - & NR \\
\hline \multirow{2}{*}{$\begin{array}{l}\text { Rhodostemonodaphne } \\
\text { crenaticupula }\end{array}$} & Folhas & - & + & $78,16 \pm 0,85$ \\
\hline & Galhos & - & + & $67,01 \pm 2,70$ \\
\hline $\begin{array}{l}\text { Rhodostemonodaphne } \\
\text { negrensis }\end{array}$ & Folhas & + & - & NR \\
\hline $\begin{array}{l}\text { Rhodostemonodaphne } \\
\text { parvifolia }\end{array}$ & Folhas & + & - & NR \\
\hline \multirow{2}{*}{$\begin{array}{l}\text { Rhodostemonodaphne } \\
\text { recurva }\end{array}$} & Folhas & + & + & $110,46 \pm 2,21$ \\
\hline & Galhos & - & + & $198,26 \pm 1,07$ \\
\hline \multirow{2}{*}{ Sextonia rubra } & Folhas & - & + & $10,043 \pm 0,317$ \\
\hline & Galhos & - & + & $8,105 \pm 1,152$ \\
\hline Padrão & & + & + & $4,13 \pm 0,042$ \\
\hline
\end{tabular}

$\mathrm{CE}_{50}=$ concentração eficiente; $\mathrm{DP}=$ desvio padrão da média; $\mathrm{NR}$ = não realizado

$\mu \mathrm{g} \cdot \mathrm{mL}^{-1}$, galhos), $M$. itauba (CE $\mathrm{CE}_{50}$ de $10,74 \mu \mathrm{g} \cdot \mathrm{mL}^{-1}$, folhas), O. minor $\left(\mathrm{CE}_{50} 8,21 \mu \mathrm{g} \cdot \mathrm{mL}^{-1}\right.$, folhas; e $9,08 \mu \mathrm{g} \cdot \mathrm{mL}^{-1}$, galhos) e $S$. rubra $\left(\mathrm{CE}_{50}\right.$ de $10,04 \mu \mathrm{g} \cdot \mathrm{mL}^{-1}$, folhas; e $8,11 \mu \mathrm{g} \cdot \mathrm{mL}^{-1}$, galhos). As espécies $O$. minor e $S$. rubra foram as únicas que apresentaram atividade em ambas as partes da planta (galhos e folhas).

Comparando com os valores obtidos para a quercetina $\left(\mathrm{CE}_{50}\right.$ de $\left.4,13 \mu \mathrm{g} \cdot \mathrm{mL}^{-1}\right)$, o extrato em etanol dos galhos de L. martiniana foi o que se comportou de forma mais semelhante ao padrão.Em alguns trabalhos sobre a composição química das espécies desse gênero relata-se a presença de lignanas furofurânicas (Alegrio et al. 1981), monoterpenos, sesquiterpenos (Maia et al. 1985) e o fenilpropanoides (Giesbrecht et al. 1974).

A análise qualitativa da atividade acetilcolinesterásica revelou que, de acordo com o método empregado, 11 extratos apresentaram resultado positivo. Destes, cinco extratos foram obtidos a partir das folhas (E. citriodora, $O$. minor, $R$. negrensis, $R$. parvifolia e $R$. recurva)e seis a partir dos galhos (A. panurensis, L. cannella angustata, L.martiniana, $O$. ceanothifolia, O. leucoxylo ne O. Minor). Dessa forma, tais espécies são candidatas a um possível estudo bioguiado 
visando investigaçóes quantitativas, assim como isolamento e elucidação estrutural das substâncias ativas.

O gênero Ocotea é um dos mais estudados na família Lauraceae e apresenta o maior número de espécies medicinais da família,com atividade antioxidante (Bruni et al. 2003)e efeito depressor do SNC (Morais et al. 1998). Considerando este gênero como o mais expressivo nos isolamentos de alcaloides de Lauraceae brasileiras (Zanin e Lordello 2007), sugere-se a possível relação da capacidade de inibição da enzima acetilcolinesterase com a presença de alcaloides.

\section{CONCLUSÕES}

Os resultados dos ensaios de atividade antioxidante não apresentaram relação direta com os resultados da inibição da enzima acetilcolinesterase, à exceção da espécie $O$. minor que foi a mais ativa, com pronunciada atividade antioxidante e inibição da enzima tanto nos extratos de galhos quanto de folhas. A maior capacidade de sequestro do radical DPPH foi observado no extrato dos galhos da espécie L. martiniana, com $\mathrm{CE}_{50}$ de $6,96 \mu \mathrm{g} \cdot \mathrm{mL}^{-1}$. Os resultados obtidos ilustram a importância da metodologia adotada neste trabalho como forma de orientação de pesquisas mais aprofundadas em apenas algumas das espécies estudadas. Resultados positivos em extratos, com ação semelhante ao padrão, mostram-se promissores quanto à presença de substâncias muitas vezes mais ativas, quando isoladas.

\section{AGRADECIMENTOS}

Os autores agradecem à FAPEAM, CAPES e CNPq pelo apoio financeiro.

\section{REFERENCIAS}

Alegrio, L. V.; Fo, R.B.; Gottlieb, O. R.; Maia, J. G. 1981. Lignans and neolignans from Licaria armeniaca.Phytochemistry, 20: 1963-1965.

Barbosa Filho, J. M.; Medeiros, K. C. P.; Diniz, M. F.; Batista, L. M., Athayde-Filho, P.F.; Silva, M. S.; da-Cunha, E. V.L.; Almeida, J. R.G. S.; Quintans-Júnior, L. J. 2006.Natural products inhibitors of the enzyme acetylcholinesterase. Revista Brasileira de Farmacognosia, 16: 258-285.

Berkov, S.; Batisda, J.; Nikova, M.; Viladomat, F.; Codina, C. 2008. Rapid TLC/CG-MS Identification of Acetylcholinesterase inhibitors in alkaloid extraxts.Phytochemical analysis. 19: 411419.

Bruni, R.; Medici, A.; Lista, A.; Fantini, C.; Muzzoli, M.; Dehesa, M.; Romagnoli, C.; Sacchetti, G. 2003. Chemical Composition and Biological Activities of Ishpingo Essential Oil, aTraditional Ecuadorian Spice from Ocotea quixos (Lam.) Kosterm. (Lauraceae) flower calices. Food chemistry, 85: 413-421.
Burger, W.C.; van der Werff, H. 1990. Flora costaricensis. Family 80, Lauraceae. Fieldiana. Botany, new series, 23: 1-129.

Chanderbali, A.S.; van der Werff, H.; Renner, S.S. 2001. Phylogeny and historical biogeography of Lauraceae: evidence from the chloroplast and nuclear genomes. Annals of the Missouri Botanical Garden, 88(1): 104-134.

Cordell, G. A.; Quirn-Beattie, M. L.; Farnsworth, N. R. 2001.The potential of alkaloids in drug discovery.Phytotherapy Research, 15: 183-205.

Cronquist, A. 1981.An Integrated System of Classification of Flowering Plants, Columbia University Press: New York. 1262 pp.

Ellman, G.L.; Courtney, K.D.; Andres, V.; Featherstone, R.M. 1961.A new and rapid colorimetric determination of acetylcholinesterase activity.Biochemical Pharmacology, 7: 88-95.

Giesbrecht, A. M.; Franca, N. C.; Gottlieb, O. R. 1974.The neolignans of Licaria canella.Phytochemistry, 13: 2285-2293.

Giordani, R. B.; Pagliosa, L. B.; Henriques, A. T.; Zuanazzi, J.A. 2008. Investigação do potencial antioxidante e anticolinesterásico de Hippeastrum (Amaryllidaceae). Quimica Nova, 31: 20422046.

Houghton, P. J.; Howes, M. J.; Lee, C. C.; Steventon, G. 2007.Uses and abuses of in vitro tests in ethnopharmacology: Visualizing an elephant. Journal of Ethnopharmacology, 110: 391-400.

Indra B.; Matsunaga K.; Hoshino O.; Suzuki M.; Ogasawara H.; Ohizumi Y. 2002. Structure-activity relationship of $( \pm)$-nantenine derivatives in a-1-adrenoreceptor activity.European Journal of Pharmacology, 437: 173-178.

Kashiwada, Y.;Aoshima, A.; Ikeshiro, Y.; Chen, Y.; Furukawa, H.; Itoigawa, M.; Fujioka, T.; Mihashi, K.; Cosentino, L. M.; MorrisNatschke, S. L.; Lee,K. 2005. Anti-HIV benzylisoquinoline alkaloids and flavonoids from the leaves of Nelumbo nucifera, and structure-activity correlations with related alkaloids. Bioorganic \& Medicinal Chemistry, 13: 443-448.

López, S.; Bastida, J.; Viladomat, F.; Codina, C. 2002. Acetylcholinesterase inhibitory activity oh some Amaryllidaceae alkaloids and Narcissus extratcts. Life Sciences, 71: 2521-2529.

Maia, J. G. S.; Ramos, L. S.; Luz, A. I. R. 1985. Estudo do óleo essencial do puxuri por cromatografia de gás/ espectrometria de massa (CG/EM). Acta Amazonica, 15: 179-183.

Marques, C.A. 2001. Importância econômica da família Lauraceae. Floresta e Ambiente. 8: 195-206.

Mensor, L. L. 2001.Screening of brazilian plant extract for antioxidant activity by the use of DPPH free radical method. Phytotherapy Research, 16: 127-128.

Morais, L. C. S. L.; Barbosa-Filho, J. M.; Almeida, R. N. 1998. Central depressant effects of reticuline extracted from Ocotea duckei in rats and mice. Journal of Ethnopharmacology, 62: 57-61.

Quik, M.e Jeyarasasingam, G. 1999. Nicotine receptor and Parkinson's disease.European Journal of Parkinson's disease.393: 223-230.

Quinet, A.; Andreata, R. H. P. 2002.Lauraceae Jussieu na Reserva Ecológica de Macaé de Cima, município de Nova Friburgo. Rio de Janeiro, Brasil. Rodriguésia, Rio de Janeiro, 53: 59pp. 
Rhee, I. K.; Van der Meent, M.; Ingkaninan, K.; Verpoorte, R. 2001.Screening for acetylcholinesterase inhibitors from from Amararyllidaceae using silica gel thin-layer chromatography in combination with bioactivity staining. Journal of Chromatography A, 915: 217-223.

Ribeiro, R.A.; Carmo, L.G.; Vladimorova, I.; Jurkiewicz, N.H.; Jurkiewicz, A. 2003. Nantenine blocks muscle contraction and $\mathrm{Ca}^{2+}$ transient induced by noradrenaline and $\mathrm{K}^{+}$in rat vas deferens. European Journal of Pharmacology, 470: 37-43.

Soler-rivas C.; Espín J. C; Wishers, H. J. (2000). An easy and fast test to compare totalfree radical scanvenger capacity of foodstuffs. Phytochem Analysis, 11: 1-9.
Traykova M.; Traykov T.; Hadjimitova V.; Krikorian K.; Bojadgieva N. 2003. Antioxidant Properties of Galantamine Hydrobromide. Verlag der Zeitschrift für Naturforschung, 58: 361-365.

Viegas Junior,; Bolzani, V. S.; Furlan, M.; Fraga, C. A. M.; Barreiro. 2004. Produtos Naturais como candidatos a fármacos úteis no tratamento do mal de Alzheimer. Química Nova, 27: 655-660.

Wagner, H.; Bladt, S. 1996. Plant drug analysis. 2 ed. New York: Springer Verlag, 318 pp.

Woo, S.H.; Sun, N.; Cassady, J. M.; Snapka, R.M. 1999. Topoisomerase II inhibition by aporphine alkaloids.Biochemical Pharmacology, 57: 1141-1145.

Zanin, S.M.W.; Lordello, A. L.L. 2007.Alcalóides aporfinóides do gênero Ocotea (Lauraceae). Quimica Nova, 30: 92-98.

Recebido em: 02/08/2011

Aceito em: 03/12/2011 